$11-2014$

Use of antenatal corticosteroids and tocolytic drugs in preterm births in 29 countries: an analysis of the WHO Multicountry Survey on Maternal and Newborn Health

Joshua P. Vogel

João Paulo Souza

A Metin Gülmezoglu

Rintaro Mori

Pisake Lumbiganon

See next page for additional authors

Follow this and additional works at: https://ecommons.aku.edu/eastafrica_fhs_mc_obstet_gynaecol

Part of the Maternal and Child Health Commons, and the Obstetrics and Gynecology Commons 


\section{Authors}

Joshua P. Vogel, João Paulo Souza, A Metin Gülmezoglu, Rintaro Mori, Pisake Lumbiganon, Zahida Qureshi, Guillermo Carroli, Malinee Laopaiboon, Bukola Fawole, and Marleen Temmerman 


\title{
Use of antenatal corticosteroids and tocolytic drugs in preterm births in 29 countries: an analysis of the WHO Multicountry Survey on Maternal and Newborn Health
}

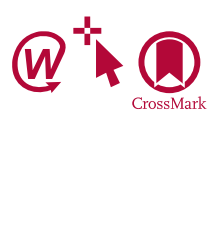

\begin{abstract}
Joshua P Vogel, João Paulo Souza, A Metin Gülmezoglu, Rintaro Mori, Pisake Lumbiganon, Zahida Qureshi, Guillermo Carroli, Malinee Laopaiboon, Bukola Fawole, Togoobaatar Ganchimeg, Jun Zhang, Maria Regina Torloni, Meghan Bohren, Marleen Temmerman, for the WHO Multi-Country Survey on Maternal and Newborn Health Research Network
\end{abstract}

\begin{abstract}
Summary
Background Despite the global burden of morbidity and mortality associated with preterm birth, little evidence is available for use of antenatal corticosteroids and tocolytic drugs in preterm births in low-income and middle-income countries. We analysed data from the WHO Multicountry Survey on Maternal and Newborn Health (WHOMCS) to assess coverage for these interventions in preterm deliveries.
\end{abstract}

Methods WHOMCS is a facility-based, cross-sectional survey database of birth outcomes in 359 facilities in 29 countries, with data collected prospectively from May 1, 2010, to Dec 31, 2011. For this analysis, we included deliveries after 22 weeks' gestation and we excluded births that occurred outside a facility or quicker than $3 \mathrm{~h}$ after arrival. We calculated use of antenatal corticosteroids in women who gave birth between 26 and 34 weeks' gestation when antenatal corticosteroids are known to be most beneficial. We also calculated use in women at 22-25 weeks' and 34-36 weeks' gestation. We assessed tocolytic drug use, with and without antenatal corticosteroids, in spontaneous, uncomplicated preterm deliveries at 26-34 weeks' gestation.

Findings Of 303842 recorded deliveries after 22 weeks' gestation, 17705 (6\%) were preterm. 3900 (52\%) of 7547 women who gave birth at 26-34 weeks' gestation, 94 (19\%) of 497 women who gave birth at 22-25 weeks' gestation, and $2276(24 \%)$ of 9661 women who gave birth at 35-36 weeks' gestation received antenatal corticosteroids. Rates of antenatal corticosteroid use varied between countries (median 54\%, range 16-91\%; IQR 30-68\%). Of 4677 women who were potentially eligible for tocolysis drugs, $1276(27 \%)$ were treated with bed rest or hydration and $2248(48 \%)$ received no treatment. $\beta$-agonists alone $(n=346,7 \%)$ were the most frequently used tocolytic drug. Only $848(18 \%)$ of potentially eligible women received both a tocolytic drug and antenatal corticosteroids.

Interpretation Use of interventions was generally poor, despite evidence for their benefit for newborn babies. A substantial proportion of antenatal corticosteroid use occurred at gestational ages at which benefit is controversial, and use of less effective or potentially harmful tocolytic drugs was common. Implementation research and contextualised health policies are needed to improve drug availability and increase compliance with best obstetric practice.

Funding UNDP-UNFPA-UNICEF-WHO-World Bank Special Programme of Research, Development and Research Training in Human Reproduction (HRP); WHO; USAID; Ministry of Health, Labour and Welfare of Japan; Gynuity Health Projects.

Copyright (C) 2014. World Health Organization. Published by Elsevier Ltd/Inc/BV. All rights reserved.

\section{Introduction}

More than 15 million infants are born preterm every year and preterm birth is the largest cause of death among newborn babies (age up to 28 days) and the second largest cause of death in children younger than 5 years. ${ }^{1}$ More than $60 \%$ of preterm deliveries occur in Africa and Asia. The deleterious effects of preterm delivery on newborn babies can be mitigated through appropriate use of proven interventions such as antenatal corticosteroids for fetal lung maturation (along with other benefits) ${ }^{2}$ and tocolytic drugs to delay delivery and potentiate the effects of antenatal corticosteroids or allow transfer to a higher-level facility before delivery. ${ }^{3}$
Injections of corticosteroids before delivery to induce fetal lung maturation and thereby prevent newborn morbidity and mortality has been comprehensively studied for nearly four decades. ${ }^{4}$ The most recent Cochrane review (2006) for use of antenatal corticosteroids in women with preterm delivery included 21 randomised controlled trials of 3885 women and 4269 babies. $^{2}$ Investigators concluded that antenatal corticosteroid use was associated with an overall $31 \%$ reduction in neonatal deaths, and significant reductions in risks of respiratory distress syndrome $(34 \%)$, cerebroventricular haemorrhage (46\%), necrotising enterocolitis (54\%), need for respiratory support or intensive-care admission (20\%) and systemic infections in the first $48 \mathrm{~h}$ of life (44\%).
Published Online August 13, 2014 http://dx.doi.org/10.1016/ S0140-6736(14)60580-8 See Online/Comment http://dx.doi.org/10.1016 50140-6736(14)60722-4

School of Population Health, Faculty of Medicine, Dentistry and Health Sciences, University of Western Australia, Crawley, WA, Australia (J P Vogel MBBS); UNDP-UNFPA-UNICEF-WHOWorld Bank Special Programme of Research, Development and Research Training in Human Reproduction, Department of Reproductive Health and Research, WHO, Geneva, Switzerland (J P Vogel, JP Souza MD, A M Gülmezoglu MD $M$ Bohren MSPH, M Temmerman MD) Department of Social Medicine, Ribeirão Preto Medical School, University of São Paulo, Ribeirão Preto, Brazil (J P Souza); Department of Health Policy, National Center for Child Health and Development, Tokyo, Japan (R Mori MD, T Ganchimeg MD); Department of Obstetrics and Gynecology, Faculty of Medicine

(Prof P Lumbiganon MD), and Department of Biostatistics and Demography, Faculty of Public Health (Prof M Laopaiboon PhD), Khon Kaen University, Khon Kaen, Thailand Department of Obstetrics and Gynaecology, School of Medicine, College of Health Science, University of Nairobi, Nairobi, Kenya (Z Qureshi MD), Centro Rosarino de Estudios Perinatales, Rosario, Argentina (G Carroli MD); University of Ibadan, Ibadan, Nigeria (B Fawole MD); Xinhua Hospital, Shanghai Jiao Tong University School of Medicine, Shanghai, China (Prof J Zhang MD); Department of Obstetrics, 
São Paulo Federal University, São Paulo, Brazil (M R Torloni MD); Brazilian Cochrane Centre, São Paulo, Brazil (M R Torloni); and Johns Hopkins Bloomberg School of Public Health, Department of Population, Family and Reproductive Health, Baltimore, MD, USA (M Bohren)

Correspondence to: Dr Joshua P Vogel, Department of Reproductive Health and Research, World Health Organization, Geneva 1211, Switzerland vogeljo@who.int

For more on World Bank's income groups see http://data. worldbank.org/about/countryand-lending-groups
Antenatal corticosteroids were effective when given from 26 weeks' to 34 weeks' plus 6 days gestation (even if given less than $24 \mathrm{~h}$ before delivery) and did not increase risk of maternal death, chorioamnionitis, or puerperal sepsis. A meta-analysis of the four randomised controlled trials for antenatal corticosteroids for preterm births in middleincome countries suggested that the reduction in mortality might be greater in these countries than in high-income countries. ${ }^{5}$ Benefit of antenatal corticosteroids outside this gestational age range is controversial; however, observational evidence suggests effectiveness when given between 22 weeks' and 26 weeks gestation, ${ }^{6}$ and investigators of one trial reported that antenatal betamethasone given to women pregnant with term infants reduced neonatal respiratory distress and admission to neonatal special-care units in newborn babies born by elective caesarean section. ${ }^{7}$ Despite the global burden of newborn morbidity and mortality related to preterm births, uptake of antenatal corticosteroids worldwide has been poor-the Bellagio Child Survival Study group ${ }^{8}$ estimated antenatal corticosteroid coverage in 2000 to be just $5 \%$ for the 42 countries that had $90 \%$ of the world's under- 5 deaths. Recent data for worldwide use of antenatal corticosteroids are not available.

Spontaneous preterm labour causes $40-45 \%$ of preterm births. ${ }^{9}$ Tocolytic drugs (such as $\beta$-agonists, calciumchannel blockers, and oxytocin antagonists) can be used as temporising measures to inhibit labour progression for up to 7 days. ${ }^{10-12}$ Use of tocolytic drugs alone has not been shown to reduce perinatal mortality (although trials have been underpowered for this outcome). ${ }^{3}$ However, use of the drugs to delay delivery is recommended to permit transfer to a higher-level facility and to potentiate the effects of corticosteroids (and hence should be used in conjunction with antenatal corticosteroids). ${ }^{11,12}$ We did not find any published reports on patterns of tocolytic drug use in preterm labour in low-income and middleincome countries, despite its importance in the management of preterm birth.

We did an analysis of the WHO Multicountry Survey of Maternal and Newborn Health (WHOMCS) dataset for more than 314000 facility-based deliveries in 29 countries. We aimed to describe patterns of use of antenatal corticosteroids in preterm deliveries and assess the use of tocolytic drugs in spontaneous preterm deliveries.

\section{Methods}

\section{Study design and participants}

WHOMCS was a cross-sectional, facility-based survey of deliveries between May 1, 2010, and Dec 31, 2011. WHOMCS aimed to characterise severe maternal, perinatal, and neonatal morbidity for a worldwide network of health facilities, with particular focus on WHO maternal near-miss indicators. ${ }^{13}$ Methodological details for WHOMCS have been described elsewhere. . $^{13,14}$ Investigators used a stratified, multistage cluster sampling approach to obtain a global sample of countries from
Africa, Asia, Latin America, and the Middle East. Within each country, the capital city was sampled, along with two randomly selected provinces (probability proportional to population). From these areas, seven facilities that had more than 1000 deliveries per year and the capacity to do caesarean sections were randomly selected (if fewer than seven facilities were available, then all were selected). All facilities that were chosen agreed to participate. Data were collected for 2 months in institutions with 6000 or more deliveries every year and for 3 months in institutions with fewer than 6000 deliveries every year. When facilities expected fewer than 3000 deliveries, the data collection period was extended to 4 months.

Study participants were all women who gave birth in participating facilities and women with a severe maternal outcome (death or near-miss) associated with pregnancy, childbirth, or puerperium. Data were obtained prospectively, from time of presentation at the facility until discharge or 7 days post partum (whichever was first). Maternal or perinatal adverse outcomes that occurred after discharge or day 7 or during a post-partum referral were not included. Data collectors reviewed medical records and abstracted de-identified data from records into the individual data form at the time of discharge, transfer, or death. An institutional data form about available obstetric and newborn services was completed in consultation with the head of the department of obstetrics for each facility. Overall, 359 facilities in 29 countries participated in WHOMCS, and data from 314623 women were recorded. For the purposes of comparison, we defined high-income countries (Japan and Qatar) and low-income and middle-income countries (the remaining 27 countries) according to the World Bank's classification for income group's WHOMCS was approved by the WHO Ethics Review Committee and relevant ethics clearance bodies in participating countries. All authors had access to the WHO Multicountry Survey database; JPV, JPS, and AMG were responsible for the decision to submit the manuscript for publication.

\section{Procedures}

WHOMCS captured information about a range of maternal sociodemographic, medical, and obstetric characteristics and outcomes for mothers and newborn babies. The gestational age was recorded in completed weeks on the basis of best available obstetric estimate; the method of estimation was not recorded. Preterm births were defined as infants born before 37 weeks of gestation. The use of antenatal corticosteroids in preterm births was recorded; however, the type, timing, number of doses, and dosing schedule was not. The use of five drug classes as tocolytic drugs for preterm labour was also recorded- $\beta$-agonists (eg, terbutaline, ritodrine), nonsteroidal anti-inflammatory drugs (NSAIDs) and cyclooxygenase (COX) inhibitors (eg, indometacin), calcium-channel blockers (eg, nifedipine), oxytocin antagonists (eg, atosiban), and magnesium sulphate. Use 


\begin{tabular}{|c|c|c|c|c|c|c|c|c|c|}
\hline & \multicolumn{3}{|c|}{$\begin{array}{l}\text { Births at } 22-25 \text { weeks' gestation } \\
(n=497)\end{array}$} & \multicolumn{3}{|c|}{$\begin{array}{l}\text { Births at } 26-34 \text { weeks' gestation } \\
(n=7547)\end{array}$} & \multicolumn{3}{|c|}{$\begin{array}{l}\text { Births at } 35-36 \text { weeks' gestation } \\
(n=9661)\end{array}$} \\
\hline & All deliveries & $\begin{array}{l}\text { Spontaneous } \\
\text { deliveries* } †\end{array}$ & $\begin{array}{l}\text { Provider- } \\
\text { initiated } \\
\text { deliveries* } \ddagger\end{array}$ & $\begin{array}{l}\text { All } \\
\text { deliveries }\end{array}$ & $\begin{array}{l}\text { Spontaneous } \\
\text { deliveriests }\end{array}$ & $\begin{array}{l}\text { Provider- } \\
\text { initiated } \\
\text { deliveries } \neq \$\end{array}$ & $\begin{array}{l}\text { All } \\
\text { deliveries }\end{array}$ & $\begin{array}{l}\text { Spontaneous } \\
\text { deliveries† } \mathbf{~}\end{array}$ & $\begin{array}{l}\text { Provider- } \\
\text { initiated } \\
\text { deliveries } \neq \mathbf{9}\end{array}$ \\
\hline Did not receive antenatal corticosteroids & $362(73 \%)$ & $235(71 \%)$ & $112(77 \%)$ & $3373(45 \%)$ & $2190(45 \%)$ & $1163(45 \%)$ & $6934(72 \%)$ & $5029(74 \%)$ & $1894(67 \%)$ \\
\hline Received antenatal corticosteroids & $94(19 \%)$ & $69(21 \%)$ & $25(17 \%)$ & $3900(52 \%)$ & $2522(51 \%)$ & $1374(53 \%)$ & $2276(24 \%)$ & $1440(21 \%)$ & $827(29 \%)$ \\
\hline Use of antenatal corticosteroids not recorded & $41(8 \%)$ & $25(8 \%)$ & $9(6 \%)$ & $274(4 \%)$ & $194(4 \%)$ & $75(3 \%)$ & $451(5 \%)$ & $349(5 \%)$ & $99(4 \%)$ \\
\hline
\end{tabular}

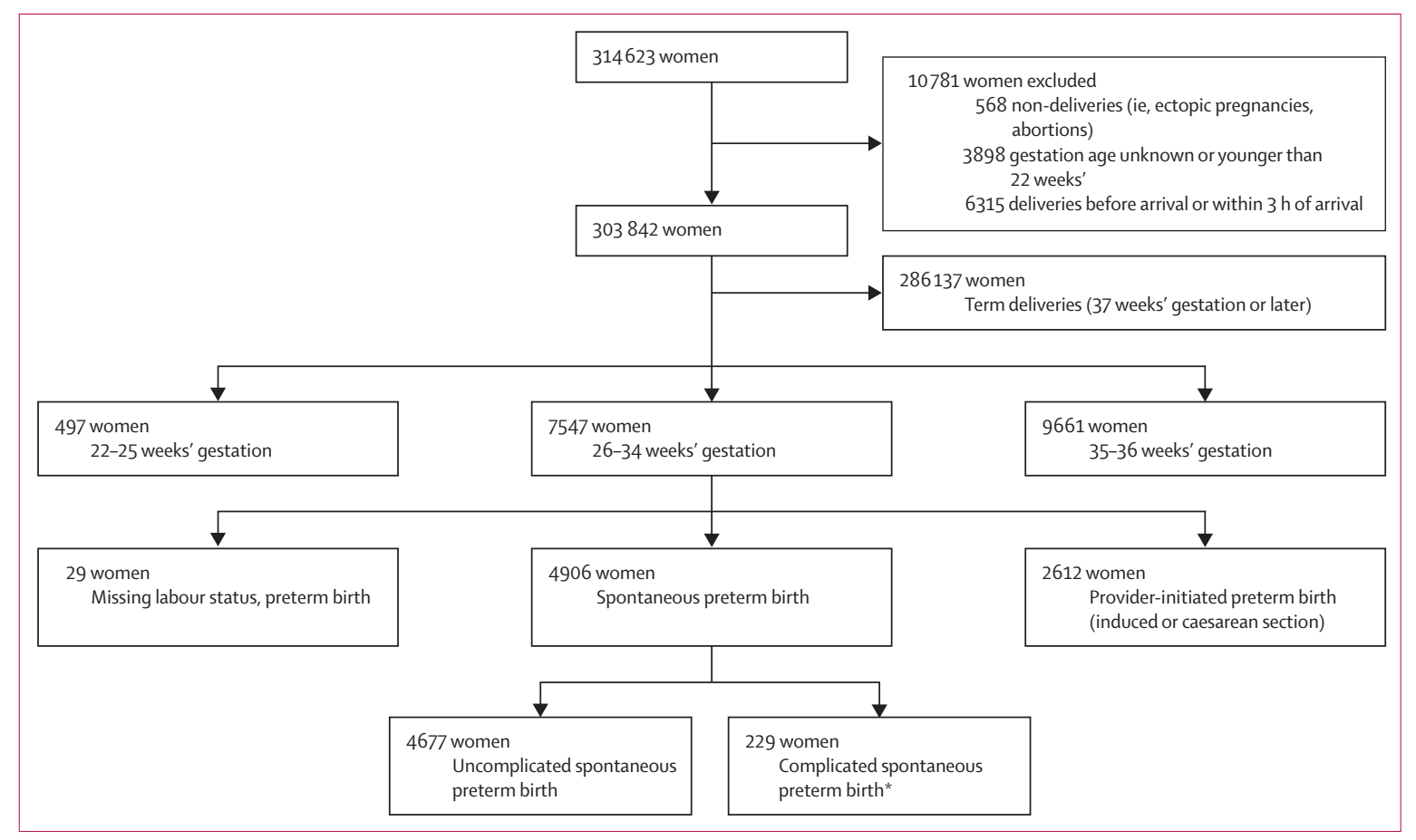

Figure 1: Population profile

*Complicated spontaneous preterm birth includes women who have eclampsia, placental abruption, or sepsis (ie, use of tocolytic drugs is contraindicated).

of bed rest, hydration, or no treatment for preterm labour was also recorded.

\section{Statistical analysis}

For our analysis, we included pregnancies at or longer than 22 weeks' gestation and excluded abortions, ectopic pregnancies, and pregnancies with unknown gestational age. We excluded deliveries that occurred before a woman's arrival at the facility or within $3 \mathrm{~h}$ of arrival because antenatal corticosteroids and tocolytic drugs would be less applicable in these contexts. Preterm deliveries were stratified into three mutually exclusive groups: 22-25 weeks', 26-34 weeks', and 35-36 weeks' gestation. We established the rate of documented antenatal corticosteroid use, both overall and for each country. We identified women who had spontaneous preterm labour at 26-34 weeks' gestation and who did not have a major contraindication to tocolysis (ie, eclampsia, placental abruption, or sepsis). We reported on the documented use of tocolysis treatments, with and without antenatal corticosteroids, in these women. We analysed the crude and adjusted associated effect of maternal, neonatal, and facility characteristics on administration of antenatal corticosteroids by using a multilevel logistic regression model. We also reported their individual effects with odds ratios, and adjusted odds ratios and their 95\% CIs. Maternal characteristics recorded were age, marital status, education level, parity, mode of delivery, presence of disorders (ie, chronic hypertension, pre-eclampsia or eclampsia, pyelonephritis, influenza-like illness, other infections or sepsis, HIV/AIDS, and malaria and dengue fever), and number 
and sex of fetuses. Facility characteristics recorded were location, level of facility, and level of services available.

We used SPSS version 20.0.0 procedure MULTIPLE IMPUTATION to impute missing values for all model variables (see missing rates in table 1). Overall missing rates were $5 \%$ or less for all variables, except for years of education $(7 \cdot 7 \%)$. Five imputed datasets were created and used in our model. We converted continuous variables (maternal age, years of education, and parity) to categorical variables after imputation for modelling. We also adjusted our model (using SPSS function GENLINMIXED) for clustering due to hierarchical design of the survey (clustering of women within facilities and facilities within countries was accounted for by use of health facilities as sampling units and countries as strata) with random intercepts at the country and facility level. Because of the weighted sampling design of the WHOMCS, our analysis was self-weighted and no further weighting was applied.

\section{Role of the funding source}

JPV, JPS, AMG, MB, and MT are employed by WHO. Some of the funds for the study were from WHO. The non-WHO sponsors of the study had no role in study design, data collection, data analysis, data interpretation, or writing of the report. All authors had access to the WHOMCS database. JPV, JPS, and AMG made the decision to submit for publication.

\section{Results}

Of 303842 women included in our analysis, 17705 (6\%) gave birth preterm (figure 1). These deliveries occurred across 359 facilities, mainly in secondary ( $N=94740,31 \%)$

\begin{tabular}{|c|c|c|c|c|c|c|}
\hline & \multicolumn{3}{|c|}{$\begin{array}{l}\text { Coverage of corticosteroids in all deliveries at } \\
26-34 \text { weeks' gestation }\end{array}$} & \multicolumn{3}{|c|}{$\begin{array}{l}\text { Coverage of both tocolytic drugs and antenatal } \\
\text { corticosteroids in uncomplicated, spontaneous } \\
\text { deliveries at 26-34 weeks' gestation }\end{array}$} \\
\hline & $\begin{array}{l}\text { Women who } \\
\text { received drugs (n) }\end{array}$ & Proportion (\%) & $95 \% \mathrm{Cl}^{*}$ & $\begin{array}{l}\text { Women who } \\
\text { received drugs (n) }\end{array}$ & Proportion (\%) & $95 \% \mathrm{Cl}^{*}$ \\
\hline Afghanistan & $48(297)$ & $16 \%$ & $6 \cdot 6-34 \cdot 5$ & $2(207)$ & $1 \%$ & $0 \cdot 2-5 \cdot 2$ \\
\hline Angola & $33(129)$ & $26 \%$ & $12 \cdot 2-46 \cdot 0$ & $2(79)$ & $3 \%$ & $0.8-7 \cdot 6$ \\
\hline Argentina & $136(185)$ & $74 \%$ & $62 \cdot 9-82 \cdot 0$ & $52(88)$ & $59 \%$ & $46 \cdot 7-70 \cdot 5$ \\
\hline Brazil & $86(288)$ & $30 \%$ & $13 \cdot 9-52 \cdot 9$ & $14(105)$ & $13 \%$ & $8 \cdot 6-20 \cdot 1$ \\
\hline Cambodia & $73(127)$ & $58 \%$ & $47 \cdot 2-67 \cdot 1$ & $9(75)$ & $12 \%$ & $3 \cdot 7-32 \cdot 8$ \\
\hline China & $158(234)$ & $68 \%$ & $58 \cdot 7-75 \cdot 2$ & $52(125)$ & $42 \%$ & $30 \cdot 0-54 \cdot 2$ \\
\hline Democratic Republic of the Congo & $26(159)$ & $16 \%$ & $6 \cdot 8-34 \cdot 4$ & $9(131)$ & $7 \%$ & $1.7-23.6$ \\
\hline Ecuador & $84(237)$ & $35 \%$ & $16 \cdot 8-59 \cdot 8$ & $34(148)$ & $23 \%$ & $9 \cdot 7-45 \cdot 3$ \\
\hline India & $1021(1481)$ & $69 \%$ & $60 \cdot 8-76 \cdot 1$ & $140(1102)$ & $13 \%$ & $7 \cdot 5-20 \cdot 7$ \\
\hline Japan & $30(52)$ & $58 \%$ & $38 \cdot 2-75 \cdot 0$ & $12(22)$ & $55 \%$ & $28 \cdot 8-78 \cdot 1$ \\
\hline Jordan & $19(21)$ & $91 \%$ & $78 \cdot 0-100$ & $5(8)$ & $63 \%$ & $29 \cdot 0-96 \cdot 1$ \\
\hline Kenya & $173(542)$ & $32 \%$ & $21 \cdot 3-44 \cdot 8$ & $23(334)$ & $7 \%$ & $3 \cdot 0-15 \cdot 0$ \\
\hline Lebanon & $43(76)$ & $57 \%$ & $41 \cdot 4-70 \cdot 6$ & $16(46)$ & $35 \%$ & $19 \cdot 9-53 \cdot 4$ \\
\hline Mexico & $300(558)$ & $54 \%$ & $38.9-68.0$ & $91(391)$ & $23 \%$ & $18 \cdot 9-28 \cdot 3$ \\
\hline Mongolia & $108(156)$ & $69 \%$ & $62 \cdot 8-75 \cdot 0$ & $17(55)$ & $31 \%$ & $23 \cdot 0-40 \cdot 1$ \\
\hline Nepal & $46(236)$ & $20 \%$ & $5 \cdot 7-49 \cdot 1$ & $0(187)$ & 0 & .. \\
\hline Nicaragua & $130(184)$ & $71 \%$ & $63 \cdot 8-76 \cdot 7$ & $56(92)$ & $61 \%$ & $45 \cdot 0-74 \cdot 7$ \\
\hline Niger & $12(60)$ & $20 \%$ & $7 \cdot 5-43 \cdot 7$ & $2(42)$ & $5 \%$ & $1 \cdot 2-17 \cdot 4$ \\
\hline Nigeria & $109(367)$ & $30 \%$ & $19 \cdot 7-42 \cdot 1$ & $27(188)$ & $14 \%$ & $5 \cdot 9-31 \cdot 1$ \\
\hline Occupied Palestinian territory & $22(25)$ & $88 \%$ & $75 \cdot 3-100$ & $4(4)$ & $100 \%$ & .. \\
\hline Pakistan & $250(398)$ & $63 \%$ & $53 \cdot 3-71 \cdot 4$ & 37 (195) & $19 \%$ & $9 \cdot 6-34 \cdot 0$ \\
\hline Paraguay & 68 (99) & $69 \%$ & $60 \cdot 3-76 \cdot 0$ & $28(56)$ & $50 \%$ & $43 \cdot 4-56 \cdot 6$ \\
\hline Peru & $302(403)$ & $75 \%$ & $67 \cdot 1-81 \cdot 4$ & $78(183)$ & $43 \%$ & $30 \cdot 9-55 \cdot 2$ \\
\hline Philippines & $120(256)$ & $47 \%$ & $37 \cdot 0-57 \cdot 0$ & $33(193)$ & $17 \%$ & $10 \cdot 5-26 \cdot 6$ \\
\hline Qatar & $9(29)$ & $31 \%$ & $14 \cdot 2-47 \cdot 8$ & $1(18)$ & $6 \%$ & $0-16 \cdot 2$ \\
\hline Sri Lanka & $287(439)$ & $65 \%$ & $56 \cdot 5-73 \cdot 3$ & $47(220)$ & $21 \%$ & $12 \cdot 9-33 \cdot 3$ \\
\hline Thailand & $108(244)$ & $44 \%$ & $34 \cdot 6-54 \cdot 3$ & $44(172)$ & $26 \%$ & $18 \cdot 8-33 \cdot 8$ \\
\hline Uganda & $41(154)$ & $27 \%$ & $19 \cdot 3-35 \cdot 5$ & $0(110)$ & $0 \%$ & .. \\
\hline Vietnam & $58(111)$ & $52 \%$ & $40 \cdot 6-63 \cdot 6$ & $13(101)$ & $13 \%$ & $5 \cdot 1-29 \cdot 1$ \\
\hline Total & $3900(7547)$ & $52 \%$ & $47 \cdot 5-55 \cdot 8$ & 848 (4677) & $18 \cdot 1 \%$ & $15 \cdot 4-21 \cdot 2$ \\
\hline
\end{tabular}




\begin{tabular}{|c|c|c|c|c|}
\hline & $\begin{array}{l}\text { Received antenatal } \\
\text { corticosteroids } \\
(\mathrm{n}=3900)\end{array}$ & $\begin{array}{l}\text { Did not receive } \\
\text { antenatal } \\
\text { corticosteroids } \\
(n=3373)\end{array}$ & $\begin{array}{l}\text { Odds ratio* } \\
(95 \% \mathrm{Cl})\end{array}$ & $\begin{array}{l}\text { Adjusted odds ratio } \dagger \\
(95 \% \mathrm{Cl})\end{array}$ \\
\hline \multicolumn{5}{|l|}{ Maternal age } \\
\hline Younger than 20 years & $332(9 \%)$ & $437(13 \cdot 0 \%)$ & $0.62(0.50-0.77)$ & $0.70(0.57-0.86)$ \\
\hline $20-35$ years & $3116(80 \%)$ & $2547(75 \cdot 5 \%)$ & Ref & Ref \\
\hline Older than 35 years & $443(11 \%)$ & $379(11 \cdot 2 \%)$ & $0.96(0.78-1.17)$ & $0.95(0.76-1.20)$ \\
\hline Data missing & $9(<1 \%)$ & $10(<1 \%)$ & .. & .. \\
\hline \multicolumn{5}{|l|}{ Marital status } \\
\hline Married & $378(10 \%)$ & $391(12 \%)$ & $0.82(0.60-1.11)$ & $1.25(0.98-1.61)$ \\
\hline Not married & $3495(90 \%)$ & $2950(88 \%)$ & Ref & Ref \\
\hline Data missing & $27(1 \%)$ & $32(1 \%)$ & .. & .. \\
\hline \multicolumn{5}{|l|}{ Years of education } \\
\hline 0 years & $573(15 \%)$ & $603(18 \%)$ & $0.64(0.41-0.99$ & $0.84(0.63-1.11)$ \\
\hline $1-6$ years & $576(15 \%)$ & $482(14 \%)$ & $0.8(0.60-1.08)$ & $0.83(0.69-0.99)$ \\
\hline $7-9$ years & $723(19 \%)$ & $660(20 \%)$ & $0.74(0.55-0.98)$ & $0.87(0.69-1.10)$ \\
\hline $10-12$ years & $1141(29 \%)$ & $875(26 \%)$ & $0.88(0.69-1.12)$ & $0.96(0.80-1.15)$ \\
\hline More than 12 years & $584(15 \%)$ & $392(12 \%)$ & Ref & Ref \\
\hline Data missing & $303(8 \%)$ & $361(11 \%)$ & .. & .. \\
\hline \multicolumn{5}{|l|}{ Parity } \\
\hline 0 & $1811(46 \%)$ & $1448(43 \%)$ & $1.06(0.95-1.19)$ & $1.20(1.07-1 \cdot 35)$ \\
\hline 1-2 children & $1580(41 \%)$ & $1343(40 \%)$ & Ref & Ref \\
\hline More than 2 children & $508(13 \%)$ & $577(17 \%)$ & $0.75(0.61-0.91)$ & $1.04(0.85-1.27)$ \\
\hline Data missing & $1(<1 \%)$ & $5(<1 \%)$ & .. & .. \\
\hline \multicolumn{5}{|l|}{ Mode of delivery } \\
\hline Vaginal delivery & $2033(52 \%)$ & $2286(68 \%)$ & Ref & Ref \\
\hline Intrapartum caesarean section & $874(22 \%)$ & $574(17 \%)$ & $1 \cdot 71(1 \cdot 34-2 \cdot 18)$ & $1.51(1.13-2.02)$ \\
\hline No-labour caesarean section & $984(25 \%)$ & $488(15 \%)$ & $2 \cdot 27(1 \cdot 77-2 \cdot 90)$ & $2.13(1.69-2.67)$ \\
\hline Data missing & $9(<1 \%)$ & $25(1 \%)$ & .. & .. \\
\hline \multicolumn{5}{|l|}{ Maternal disorders } \\
\hline Chronic hypertension & $114(3 \%)$ & $64(2 \%)$ & $1.56(1.01-2 \cdot 41)$ & $1.14(0.83-1.57)$ \\
\hline Pre-eclampsia or eclampsia & $714(18 \%)$ & $441(13 \%)$ & $1.49(1.22-1.82)$ & $1.14(0.89-1 \cdot 46)$ \\
\hline Pyelonephritis & $23(1 \%)$ & $9(<1 \%)$ & $2 \cdot 22(1 \cdot 16-4 \cdot 23)$ & $1.90(1.07-3.37)$ \\
\hline Influenza-like illness & $20(1 \%)$ & $7(<1 \%)$ & $2 \cdot 25(1.03-5.98)$ & $1.56(0.92-2.63)$ \\
\hline Other systemic infections or sepsis & $85(2 \%)$ & $61(2 \%)$ & $1.21(0.81-1 \cdot 80)$ & $1.28(0.61-2.69)$ \\
\hline HIV/AIDS or HIV wasting syndrome & $21(1 \%)$ & $28(1 \%)$ & $0.65(0.31-1 \cdot 37)$ & $1.59(0.73-3.44)$ \\
\hline Malaria or dengue fever & $16(<1 \%)$ & $27(1 \%)$ & $0.51(0.24-1.11)$ & $0.90(0.35-2.26)$ \\
\hline \multicolumn{5}{|l|}{ Sex of infant } \\
\hline Female & $1796(46 \cdot 1 \%)$ & $1592(47 \%)$ & $0.93(0.85-1.03)$ & $0.98(0.90-1.06)$ \\
\hline Male & $2096(53 \cdot 7 \%)$ & $1736(52 \%)$ & Ref & Ref \\
\hline Data missing & $8(0.2 \%)$ & $45(1 \%)$ & .. & .. \\
\hline \multicolumn{5}{|l|}{ Number of newborn babies } \\
\hline One (singleton pregnancy) & $3574(92 \%$ & $3153(94 \%)$ & Ref & Ref \\
\hline Two (twin pregnancy) & $296(8 \%)$ & $197(6 \%)$ & $1 \cdot 33(1.08-1 \cdot 63)$ & $3.59(1 \cdot 15-11 \cdot 24)$ \\
\hline Three or more (higher-order multiple pregnancy) & $29(1 \%)$ & $6(<1 \%)$ & $4 \cdot 26(1.85-9 \cdot 84)$ & $1.28(1.03-1 \cdot 60)$ \\
\hline Missing data & $1(<1 \%)$ & $17(1 \%)$ & .. & .. \\
\hline \multicolumn{5}{|l|}{ Location $\ddagger$} \\
\hline Urban & $3483(89 \%)$ & $2762(82 \%)$ & Ref & Ref \\
\hline Peri-urban & $162(4 \%)$ & $358(11 \%)$ & $0.36(0.17-0.77)$ & $0.79(0.58-1.07)$ \\
\hline Rural & $57(2 \%)$ & $96(3 \%)$ & $0.47(0.29-0.77)$ & $0.88(0.77-1.01)$ \\
\hline \multirow[t]{2}{*}{ Data missing } & $198(5 \%)$ & $157(5 \%)$ & .. & .. \\
\hline & & & \multicolumn{2}{|c|}{ (Table 3 continues on next page } \\
\hline
\end{tabular}




\begin{tabular}{|c|c|c|c|c|}
\hline & $\begin{array}{l}\text { Received antenatal } \\
\text { corticosteroids } \\
(n=3900)\end{array}$ & $\begin{array}{l}\text { Did not receive } \\
\text { antenatal } \\
\text { corticosteroids } \\
\mathrm{n}=3373 \text { ) }\end{array}$ & $\begin{array}{l}\text { Odds ratio* } \\
(95 \% \mathrm{Cl})\end{array}$ & $\begin{array}{l}\text { Adjusted odds ratio } † \\
(95 \% \mathrm{Cl})\end{array}$ \\
\hline \multicolumn{5}{|c|}{ (Continued from previous page) } \\
\hline \multicolumn{5}{|l|}{ Level of facility $\ddagger$} \\
\hline Primary & $52(1 \%)$ & $82(2 \%)$ & $0.41(0.22-0.78)$ & $0.79(0.52-1.22)$ \\
\hline Secondary & $663(17 \%)$ & $969(29 \%)$ & $0.45(0.30-0.66)$ & $0.93(0.69-1.26)$ \\
\hline Tertiary & $2677(69 \%)$ & $1743(52 \%)$ & Ref & Ref \\
\hline Other referral level & $309(8 \%)$ & $383(11 \%)$ & $0.53(0.26-1.05)$ & $1.03(0.82-1.29)$ \\
\hline Data missing & $199(5 \%)$ & $196(6 \%)$ &.. & .. \\
\hline \multicolumn{5}{|c|}{$\begin{array}{l}\text { Columns might not add to } 100 \% \text { because of rounding error. } 274 \text { women had information missing on receipt of antenatal corticosteroids and are not included. Ref=reference } \\
\text { comparator group. *Calculation of crude odds ratios adjusted for clustering due to survey design only. †Multilevel logistic regression model adjusted for maternal } \\
\text { characteristics (age, marital status, education, parity, and mode of delivery), maternal disorders (chronic hypertension, pre-eclampsia or eclampsia, pyelonephritis, } \\
\text { influenza-like illness, other infections or sepsis, HIV/AIDS, malaria, and dengue fever), infant characteristics (sex and number of fetuses), and facility characteristics (location } \\
\text { and level of facility, provision of care free of charge at facility, and level of services available at facility), as well as clustering due to survey design. } ¥ \text { As reported by head of } \\
\text { obstetrics department or facility. }\end{array}$} \\
\hline
\end{tabular}

See Online for appendix

\begin{tabular}{|lc}
\hline & $\begin{array}{l}\text { Number } \\
\text { of women } \\
(\%)\end{array}$ \\
\hline Women receiving drugs for tocolysis & $970(21 \%)$ \\
Calcium-channel blocker only & $271(6 \%)$ \\
Oxytocin antagonist only & $26(1 \%)$ \\
ß-agonists only & $346(7 \%)$ \\
NSAIDs or COX inhibitors only & $48(1 \%)$ \\
Magnesium sulphate only & $85(2 \%)$ \\
More than one drug given* & $194(4 \%)$ \\
\hline No drug given; treated with bed rest and hydration only ${ }^{*}$ & $1276(27 \%)$ \\
No treatment given for tocolysis & $2248(48 \%)$ \\
\hline Data missing & $183(4 \%)$ \\
\hline $\begin{array}{l}\text { Data for } 4677 \text { deliveries. NSAIDs=non-steroidal anti-inflammatory drugs. } \\
\text { COX=cyclo-oxygenase. }{ }^{*} \text { Use of two or more tocolytic drugs including those listed. } \\
\text { †No documented use of any of the named drugs or treatments. }\end{array}$ \\
\hline Table 4: Use of tocolytic drugs in women with uncomplicated, \\
spontaneous preterm deliveries at 26-34 weeks' gestation
\end{tabular}

and tertiary $(\mathrm{N}=131835,43 \%)$ facilities, with the rest in primary $(\mathrm{N}=16611,6 \%)$ and other referral level $(\mathrm{N}=36460$ $12 \%)$ facilities; data were missing for $8 \cdot 0 \%(\mathrm{~N}=24196)$ of deliveries. The 7547 women who gave birth at 26-34 weeks' gestation accounted for $2 \cdot 5 \%$ of all deliveries. Of these events, 4906 (65\%) women had a spontaneous preterm birth, of which 4677 (95\%) were spontaneous preterm births without a major contraindication to tocolysis. Table 1 shows data for antenatal corticosteroid use in the 17705 women who delivered preterm; 766 (4\%) had data missing for use of antenatal corticosteroids. Of the women who delivered at 26-34 weeks' gestation, 52\% received antenatal corticosteroids (table 1 ).

When stratified by spontaneous preterm birth or provider-initiated preterm birth (ie, induced labour or a pre-labour caesarean section), antenatal corticosteroid use for women at 26-34 weeks' gestation was similar $\left(\chi^{2}=0 \cdot 62\right)$. When analysed for countries, median antenatal corticosteroid coverage was 54\% (range 16-91\%, IQR 30-68\%; table 2, appendix). Only two countries exceeded $80 \%$ useJordan (91\%) and occupied Palestinian territory (88\%)however, the number of preterm babies in both countries was quite small. Although we did not know the vital status of the fetus at onset of labour, we did a sensitivity analysis by excluding the 1957 women who had stillbirth with signs of maceration (on the assumption that these fetuses were probably not alive at the start of labour). Use of antenatal corticosteroids increased from $52 \%$ ( $n=3900$ of 7547$)$ to $56 \%$ ( $n=3836$ of 6909 ) after we excluded these deliveries. Results from the multilevel logistic regression model with multiple imputation (table 3) showed that the adjusted odds of receipt of antenatal corticosteroids were significantly raised in nulliparous women and in both intrapartum caesarean and pre-labour caesarean deliveries. Women with pyelonephritis had raised adjusted odds of antenatal corticosteroid receipt, as did those pregnant with twins and higher-order multiple pregnancies. The adjusted odds of antenatal corticosteroid use were lower in women younger than 20 years and women with 1-6 years of education. The same model without multiple imputation of missing values returned similar results (data not shown), with no change in significance for covariates except for pyelonephritis (not significant in model without multiple imputation; adjusted odds ratio $1 \cdot 39,95 \% \mathrm{CI}$ $0 \cdot 79-2.43$ ) and influenza (significant in model without multiple imputation; $2 \cdot 48,1 \cdot 56-3 \cdot 93)$.

Among the 4677 women with uncomplicated, spontaneous preterm labour who were eligible for tocolytic treatment, almost half received no treatment and a quarter received non-drug treatments (table 4). The most frequently used drugs for tocolysis were $\beta$-agonists and calcium-channel blockers. Only 848 (18\%) women in this group received a tocolytic drug in 


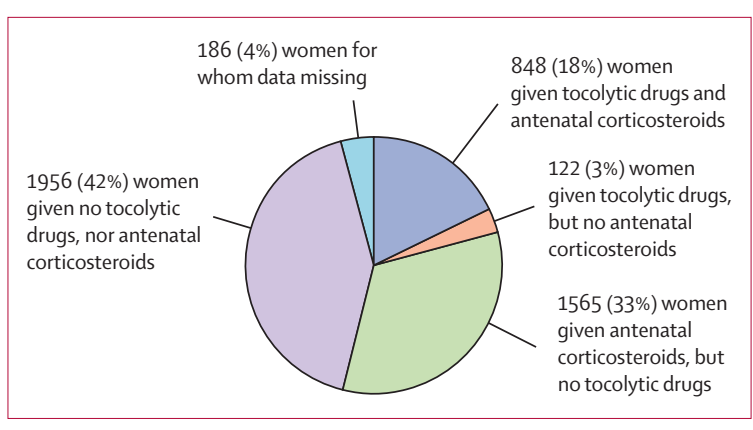

Figure 2: Use of tocolytic drugs, with and without antenatal corticosteroids, in uncomplicated spontaneous preterm births (26-34 weeks' gestation; $n=4677$ ) Tocolytic drugs for preterm labour include calcium-channel blockers, oxytocin antagonists, $\beta$-agonists, non-steroidal anti-inflammatory drugs and cyclooxygenase inhibitors, and magnesium sulphate. The no tocolytic drug group indicates women treated with bed rest, hydration, or no treatment for preterm labour.

combination with antenatal corticosteroids (figure 2). Most women received neither a tocolytic drug nor antenatal corticosteroids, or antenatal corticosteroids without a tocolysis drug; and a few received tocolysis without concurrent antenatal corticosteroids. At the country level, rates of tocolytic drug use in combination with antenatal corticosteroids were generally lowmedian rate was 19\% (range $0-100$; table 2 , appendix).

\section{Discussion}

Our analysis showed that antenatal corticosteroids and tocolytic drugs were substantially underused in women in whom they would have been beneficial. Use of antenatal corticosteroids at gestational ages at which benefit is more controversial, or use of tocolytic drugs that are ineffective or have higher rates of adverse outcomes, was common and exposed women and their babies to unnecessary risk. Nearly half of eligible women overall did not receive antenatal corticosteroids, and, in many countries, most eligible women did not receive antenatal corticosteroids. Lower-income countries such as Afghanistan, Democratic Republic of the Congo, Nepal, and Niger have particularly high rates of neonatal mortality ${ }^{15}$ and low use of antenatal corticosteroids. Antenatal corticosteroid use in the two high-income countries that we assessed (Japan 56\% and Qatar 31\%) was surprisingly low; however, this finding must be interpreted cautiously because the number of women (52 and 29, respectively) was quite small. Although there are few reports of antenatal corticosteroid use in low-income and middle-income countries (panel), other investigators have noted similarly wide variations-eg, 4-71\% of women received corticosteroids in several Latin American countries ${ }^{16-20}$ and $9-73 \%$ of women in four southeast Asian countries. ${ }^{21}$ Of perhaps greater concern is that use of antenatal corticosteroids was low even in preterm births after induction or pre-labour caesarean section; deliveries in which the timing of delivery and need for antenatal corticosteroids could be reasonably anticipated. Although we were unable to explore specific reasons to explain this finding, it suggests that barriers to poor use of antenatal corticosteroids probably extend beyond poor identification of women at risk of preterm delivery. Almost a quarter of women who delivered at 35-36 weeks' gestation received antenatal corticosteroids despite the fact that perinatal morbidity and mortality benefit at these gestational age intervals is somewhat controversial. ${ }^{2}$ Uncertainty about gestational age is common in low-income and middle-income countries because antenatal care participation and ultrasound availability can be poor-this uncertainty might have contributed to use of antenatal corticosteroids in gestational ages outside of the 26-34 weeks' gestation range. More appropriate prescribing practices could improve outcomes for the $45 \%$ of women at 26-34 weeks' gestation who did not receive antenatal corticosteroids.

The use of tocolytics was generally poor, but it is of great concern that more than a third of eligible women received ineffective treatments for preterm labour. No evidence exists to support the use of bed rest ${ }^{22}$ and hydration (if not dehydrated) ${ }^{23}$ to prevent preterm delivery, and magnesium sulphate is not effective to delay birth or prevent preterm birth. ${ }^{24}$ Haas and colleagues ${ }^{3}$ did a systematic review and network meta-analysis for drug treatment options to prevent preterm delivery and reported that prostaglandin inhibitors and calcium-channel blockers have the highest probability of improving neonatal outcomes by delaying delivery and have the lowest maternal side-effects. Prostaglandin inhibitor use is complicated by the risk of oligohydramnios and premature closure of the fetal ductus arteriosus..$^{25}$ Nifedipine is comparatively cheaper, ${ }^{26}$ is simple to administer, and is the only drug for tocolysis in the WHO Essential Medicines List. ${ }^{27} \beta$-agonists have similar effectiveness to calcium-channel blockers, ${ }^{11}$ but they have higher rates of maternal adverse effects (such as chest pain, breathing difficulties, heart irregularities, headaches, and shaking). In our analysis, $\beta$-agonists were the most commonly used effective tocolytic drug, despite the known maternal side-effects, and only $6 \%$ of eligible women received calcium-channel blockers. This pattern might be due to historical reasons; however, it shows that changes in tocolytic prescribing practices could improve both maternal and neonatal outcomes. Ideally, women in spontaneous preterm labour between 26 and 24 weeks of gestation who receive tocolytics should recieve antenatal corticosteroids in combination, yet only $15 \%$ of eligible women received both treatments and $42 \%$ received neither. As far as we know, our data are the first international comparison of tocolytic drug use with antenatal corticosteroids in preterm birth across lower-income countries. Further research on the determinants of tocolytic prescription would help to identify interventions to improve compliance with best practices in preterm labour management.

A 2013 survey by Aleman and colleagues ${ }^{28}$ identified several barriers to improved uptake of antenatal 
Panel: Research in context

\section{Systematic review}

In women at 26-34 weeks' gestation at risk of preterm birth, the efficacy of antenatal corticosteroids to accelerate fetal lung maturation is unquestioned; contributors to a 2006 Cochrane review ${ }^{2}$ explored the subject extensively. Similarly, the efficacy and adverse effects of individual tocolytic drugs (such as betamimetics, ${ }^{10}$ calcium-channel blockers, ${ }^{11}$ oxytocin receptor antagonists, ${ }_{12}^{12}$ and magnesium sulphate ${ }^{24}$ ) have been the subject of Cochrane reviews.

\section{Interpretation}

Despite the wealth of trial evidence about the efficacy and indications for antenatal corticosteroids and tocolytic drugs in preterm birth, remarkably little data are available for clinical patterns of their use, particularly in low-income and middle-income countries where most of the global burden of preterm-associated neonatal mortality occurs. Our findings provide the first internationally comparable, comprehensive study of prevalence of use for antenatal corticosteroids. We suggest that a substantial amount of preterm-birth-associated newborn morbidity and mortality could be avoided through improvements in the delivery of these known interventions. Intensive efforts to scale up use of antenatal corticosteroids in facility settings are needed. to increased use. Interventions to increase demand could also change practice. For example, community mobilisation and provision of antenatal corticosteroids free of charge to all women at risk of preterm labour would not only increase demand, but would also address the socioeconomic inequities in antenatal corticosteroid use and improve coverage in higher-risk subpopulations (such as adolescents and poorly educated mothers).

Some limitations could have affected the results of our analysis. WHOMCS was mainly a study of maternal and perinatal morbidity and mortality, of which preterm birth is an important component, but was not the survey's main aim. The number of countries participating in the multicountry survey was limited by financial constraints; hence, Europe was not sampled. Although robust, the multistage facility sampling design could potentially introduce bias, because women from countries with smaller populations are oversampled compared with women from countries with larger populations. Within the same country, when smaller, lower-level facilities are randomly sampled, complicated deliveries might be under-represented. Conversely, in countries where maternity care is centralised in a few, large facilities (particularly in the capital city) or where such facilities were randomly sampled, complicated deliveries are probably over-represented. These data are probably not representative of smaller facilities and communities not included in the sampling frame; however, we believe it is reasonable to assume that antenatal corticosteroid and tocolytic drug availability is high in large facilities, and that higher-level facilities and coverage rates are probably poorer in lower-level facilities. In some countries, the number of deliveries in lower-level facilities is proportionally greater, thus coverage might still be poor even when the national rate of facility delivery is high. Data for WHOMCS were abstracted from hospital records; in many facilities, these records are suboptimum and use of interventions might not have been documented correctly (particularly those occurring before facility admission). This bias was minimised as much as possible because data collectors consulted with medical staff about missing information; however, some treatment might be undocumented or could have occurred before arrival at the facility, leading to a possible underestimation of coverage. Conversely, failure to recognise preterm births due to poor estimation of gestational age might lead to an overestimate of drug use. Under-recognition of prematurity also probably contributed to the recorded preterm birth rate $(5 \cdot 8 \%)$ being lower than was expected based on recent estimates. ${ }^{1}$ Fetal status on arrival for delivery, as well as the type, timing, dose, and frequency of antenatal corticosteroids and tocolytic drugs, was not included in WHOMCS, and we were unable to account for all contraindications to these drugs (such as drug allergies, maternal cardiac diseases, and advanced cervical dilatation). A small proportion of antenatal corticosteroid use at 35-36 weeks' 
gestation might be attributable to women who were treated earlier than 35 weeks' gestation but who delivered after 35 weeks' during the same admission. Although missing data might have led to a bias in our findings, missing rates were generally low $(\leq 5 \%)$ and results from modelling, both with and without multiple imputation, were similar, increasing our confidence that missing values had negligible effect.

Despite the evidence for effectiveness of antenatal corticosteroids and tocolytic drugs in preterm deliveries, their use was highly variable and often poor. A substantial proportion of antenatal corticosteroid use was at extremes of gestational age when benefit is controversial, whereas a significant proportion of mothers of infants at 26-34 weeks' gestation did not receive this life-saving intervention. The use of ineffective, less effective, or potentially harmful treatments for tocolysis was also widespread. Implementation research and contextualised health policies are needed to improve drug availability and compliance with best obstetric practices.

\section{Contributors}

JPV and JPS conceptualised the Article and analysis plan. JPV did the analyses, in collaboration with JPS and AMG. All authors contributed to the interpretation of results. JPV drafted the initial report and JPS, AMG, RM, PL, ZQ, GC, ML, BF, TG, JZ, MRT, MB, and MT contributed to the content and revisions.

\section{Declaration of interests}

We declare no competing interests.

\section{Acknowledgments}

We thank all members of the WHO Multicountry Survey on Maternal and Newborn Health Research Network, including regional and country coordinators, data collection coordinators, facility coordinators, data collectors, and all staff of participating facilities who made the survey possible. JPV is supported by the Australian Postgraduate Award and the A \& A Saw Scholarship. This manuscript represents the views of the named authors only.

\section{References}

1 March of Dimes. PMNCH, Save the Children, WHO. Born Too Soon: the Global Action Report on Preterm Birth. Geneva: World Health Organization, 2012.

2 Roberts D, Dalziel S. Antenatal corticosteroids for accelerating fetal lung maturation for women at risk of preterm birth. Cochrane Database Syst Rev 2006; 3: CD004454.

3 Haas DM, Caldwell DM, Kirkpatrick P, McIntosh JJ, Welton NJ. Tocolytic therapy for preterm delivery: systematic review and network meta-analysis. BMJ 2012; 345: e6226.

$4 \quad$ Liggins GC, Howie RN. A controlled trial of antepartum glucocorticoid treatment for prevention of the respiratory distress syndrome in premature infants. Pediatrics 1972; 50: 515-25.

5 Mwansa-Kambafwile J, Cousens S, Hansen T, Lawn JEJ. Antenatal steroids in preterm labour for the prevention of neonatal deaths due to complications of preterm birth. Int J Epidemiol 2010; 39 (suppl 1): i122-33.

6 Mori R, Kusuda S, Fujimura M. Antenatal corticosteroids promote survival of extremely preterm infants born at 22 to 23 weeks of gestation. J Pediatr 2011; 159: 110-14.

7 Stutchfield P, Whitaker R, Russell I, for the Antenatal Steroids for Term Elective Caesarean Section (ASTECS) Research Team. Antenatal betamethasone and incidence of neonatal respiratory distress after elective caesarean section: pragmatic randomised trial. BMJ 2005; 331: 662.

8 Jones G, Steketee RW, Black RE, Bhutta ZA, Morris SS, for the Bellagio Child Survival Study Group. How many child deaths can we prevent this year? Lancet 2003; 362: 65-71.
9 Goldenberg RL, Culhane JF, Iams JD, Romero R. Epidemiology and causes of preterm birth. Lancet 2008; 371: 75-84.

10 Anotayanonth S, Subhedar NV, Garner P, Neilson JP, Harigopal S. Betamimetics for inhibiting preterm labour. Cochrane Database Syst Rev 2004; 4: CD004352.

11 King JF, Flenady VJ, Papatsonis DN, Dekker GA, Carbonne B. Calcium channel blockers for inhibiting preterm labour. Cochrane Database Syst Rev 2007; 1: CD002255.

12 Papatsonis D, Flenady V, Cole S, Liley H. Oxytocin receptor antagonists for inhibiting preterm labour. Cochrane Database Syst Rev 2005; 3: CD004452.

13 Souza JP, Gülmezoglu AM, Vogel J, et al. Moving beyond essential interventions for reduction of maternal mortality (the WHO Multicountry Survey on Maternal and Newborn Health): a cross-sectional study. Lancet 2013; 381: 1747-55.

14 Souza JP, Gülmezoglu AM, Carroli G, Lumbiganon P, Qureshi Z, for the WHOMCS Research Group. The World Health Organization Multicountry Survey on Maternal and Newborn Health: study protocol. BMC Health Serv Res 2011; 11: 286.

15 Requejo J, Bryce J, Victora C. Countdown to 2015: building a future for women and children. Washington DC: World Health Organization and UNICEF, 2012. http://www.countdown2015mnch.org/ documents/2012Report/2012-Complete.pdf (accessed June 12, 2014).

16 Forteza C, Diaz Rossello JL, Matijasevich A, Barros F. Morbidity and mortality of very low birth weight (VLBW) infants in Montevideo, Uruguay. Paediatr Res 2002; 77: 313-20.

17 Silva LK, Costa TP, Reis AF, Iamada NO, Azevedo AP Albuquerque CP. Assessment of quality of obstetric hospital care: use of corticoid in preterm labor. Cad Saude Publica 1999; 15: 817-29.

18 Vallejo Valdivieso N, Chinga Sampedro J, Sanchez Macias M, Tumbaco Garcia R. Epidemiologia del parto pretermino y su repercusion en la morbi-mortalidad neonatal registrados en el hospital Dr. Verdi Cevalholos Balda/Epidemiologia of the childbirth preterm and its repercussion in neonatal morbi-mortality registered in hospital Dr Verdi Cevallos. Medicina (Guayaquil) 2002; 8: 36-41.

19 Colomar M, Belizán M, Cafferata MLM, et al, for the Grupo de Estudio de Prácticas Perinatales. Practices of maternal and perinatal care performed in public hospitals of Uruguay. Ginecol Obstet Mex 2004; 72: 455-65.

20 Riganti AA, Cafferata ML, Althabe F, et al. Use of prenatal corticosteroids for preterm birth in three Latin American countries. Int J Gynaecol Obstet 2010; 108: 52-57.

21 Pattanittum P, Ewens MR, Laopaiboon M, Lumbiganon P, McDonald SJ, Crowther CA, for the SEA-ORCHID Study Group. Use of antenatal corticosteroids prior to preterm birth in four south east Asian countries within the SEA-ORCHID project. BMC Pregnancy Childbirth 2007; 8: 47.

22 Sosa C, Althabe F, Belizan J, Bergel E. Bed rest in singleton pregnancies for preventing preterm birth. Cochrane Database Syst Rev 2003; 1: CD003581.

23 Stan C, Boulvain M, Pfister R, Hirsbrunner-Amagbaly P. Hydration for treatment of preterm labour. Cochrane Database Syst Rev 2013; 11: CD003096.

24 Crowther CA, Hiller JE, Doyle LW. Magnesium sulphate for preventing preterm birth in threatened preterm labour. Cochrane Database Syst Rev 2006; 4: CD001060.

25 Goldenberg RL. The management of preterm labor. Obstet Gynecol 2002; 100: 1020-37.

26 Hayes EE, Moroz LL, Pizzi LL, Baxter JJ. A cost decision analysis of 4 tocolytic drugs. Am J Obstet Grnecol 2007; 197: 383.e1-6.

27 WHO. WHO model lists for essential medicines. Geneva: World Health Organization, 2011. http://apps.who.int/iris/bitstream/10665/93142/1/ EML_18_eng.pdf?ua=1 (accessed June 12, 2014).

28 Aleman A, Cafferata MLM, Gibbons L, et al. Use of antenatal corticosteroids for preterm birth in Latin America: providers knowledge, attitudes and practices. Reprod Health 2013; 10: 4.

29 WHO. OptimizeMNH: WHO recommendations on optimizing health worker roles to improve access to key maternal and newborn health interventions through task shifting. Geneva: World Health Organization, 2012. 